Research

Open Access

\title{
Developing an Effective Food Supplement for the Prevention of Osteoporosis
}

\section{Ping-chung LEUNG ${ }^{1,2}$, Erik Chun-hay $\mathrm{KO}^{2}$, Sammy Wing-sum SIU ${ }^{2}$, Ellie Suet-yee PANG $^{2}$, King-fai CHENG ${ }^{2}$, Yat-heung CHAN ${ }^{1}$}

${ }^{1}$ Jockey Club Centre for Osteoporosis Care and Control, The Chinese University of Hong Kong, HKSAR; ${ }^{2}$ Institute of Chinese Medicine, The Chinese University of Hong Kong, HKSAR

Corresponding author: P.C. Leung, MD, Professor, Director, Center for Clinical Trials on Chinese Medicine, The Chinese University of Hong Kong, Prince of Wales Hospital Shatin, NT, Hong Kong SAR

Submission date: July 18, 2011; Acceptance date: September 28, 11; Publication date: September 29, 2011

\section{$\underline{\text { Abstract }}$}

Background: Aging is accompanied by a gradual loss of bone mineral contents which might lead to a higher rate to fractures. In the past twenty years, many pharmaceuticals are created to prevent bone loss. Most effective drugs only suppress bone resorption, the result of which is a loss of the normal balance of bone metabolism. Anti-resorptive drugs have already shown complications arising from the loss of balanced metabolism. Therefore it becomes clear now that prevention of bone mineral loss through a natural maintenance process will be more beneficial for those not under special risks. Drug treatment could be researched for those who already developed severe osteoporosis. There are many medicinal herbs in Chinese Medicine known to be supporting bone health. Three of those herbs, Herba Epimedii, Fructus Ligustri Lucidi and Fructus Psoralea (ELP) were chosen to make a formula for the prevention of osteoporosis.

Result: One hundred and fifty postmenopausal osteopenic women were recruited, randomized and allocated to the herbal group (ELP) or placebo group. After 12 months of consumption of herbal formula, bone mineral maintenance was found when the bone mineral density (BMD) of the femur and tibia were as assessed using Dual-energy X-ray 
Absorptiometry (DEXA) and Peripheral Quantitative Computed Tomography (pQCT) machines. No adverse effects were found.

Conclusion: The clinical study using ELP was designed as a randomized controlled trial to test the efficacy of the agent used. The results showed positive support on the BMD of all bone tested. However statistical difference between the herbal and placebo group reached the significant level only in the proximal tibia and those who experienced menopause for more than ten years. This finding well indicates that ELP is good for preventive but does not satisfy the demand of a treatment agent. ELP could be recommended to those under the early threat of osteoporosis, i.e. osteopenia, to be used as a preventive agent.

Keywords: Osteoporosis, post-menopause women, herbal supplement, functional food.

\section{Background:}

Osteoporosis has become a major health issue worldwide, it is defined as a condition characterized by low bone mineral density (BMD) and micro architectural deterioration of bone tissues leading to enhanced bone fragility and a consequent increase in fracture risk [1, 2]. Postmenopausal osteoporosis typically affects women within 15-20 years after menopause. It is complicated with fractures occurring at sites that contain relatively large amounts of cancellous bone. The prevalence of osteoporotic fracture is extremely high. In the United States for women at 50 years of age the lifetime risk is $17.5 \%$ for the hip fracture, $16 \%$ for vertebral fracture, and 16\% for Colles' fracture. Approximately 50 percent of 70 -year-old women or 40 percent after the menopause will experience one or more fractures $[3,4]$.

In China, epidemiological studies have shown that the incidence of osteoporosis in the population over the age of 40 years was $16.1 \%$, over 60 years $22.6 \%$, and over 80 years as high as $50 \%$. Osteoporosis in the spine not only leads to fractures, but would also produce pain and back deformities, which seriously affect patients' quality of life [5, 6].

Estrogen plays an important role in coordinating activities of the bone-forming osteoblasts and bone-resorbing osteoclasts in bone homeostasis. Ovarian hormonal deficiency is one of the most important factors leading to postmenopausal osteoporosis, so that hormonal replacement therapy (HRT) has been popular in the early days for the prevention of bone loss in postmenopausal women [7, 8]. However, treatment with estrogen has well-known side effects such as breast soreness and nausea, and in the long-term, may lead to increased risks of breast and uterine cancer development and also venous thrombosis [9].

Subsequently, when other therapeutic measures are developed to correct the loss of 
$\mathrm{BMD}$, the same concept of quick correction, i.e. treatment, is adopted. This concept is acceptable when the loss of BMD is sharp and severe, under which circumstance, effective restoration is required. However, when the BMD is only starting to decline, there is no necessity of bringing it up artificially. It might be more favorable just to maintain the bone mineral contents and prevent any uncontrolled decline. Food supplement therefore, could be a valuable solution.

\section{Methods:}

\section{The herbal formula}

Many herbs used in traditional Chinese Medicine are known to be effective in the maintenance of health for the elderly who complain about back pain, limb pain and loss of body height. Such symptoms are known to be manifestations of "Kidney deficiency". Those herbs are used either as food or as components of complex herbal formulae.

Three herbs well known to be good for "Kidney deficiency" were selected from a list of herbs recommended in Chinese Medicine; these herbs have been used as food or components of medicinal herbal formulae. They were Herba Epimedii, Fructus Ligustri Lucidi and Fructus Psoralea (ELP). The herbs were mixed in the ratio 10:8:2 and were boiled and extracted into powder form.

Instead of using ELP immediately as a medicinal agent for trial, they were first tested in the laboratory to establish basis for scientific evidence of their bone supportive activities. The anabolic effects of the water extract of ELP on bone formation were evaluated using cultured rat osteoblast-like osteosarcoma cell line UMR-106 and rat mesenchymal stem cells (MSCs). To test for bone resorption, RAW 264.7 cell line was used to investigate the inhibition of osteoclast formations. A dosing effect with several concentrations $(1 \mathrm{~g} / \mathrm{day}, 0.5$ $\mathrm{g} /$ day and $0.175 \mathrm{~g} / \mathrm{day}$ ) of the anti-osteoporosis function of the water extract of ELP formula were tested in ovariectomy-and-calcium deficiency-induced osteoporotic rats [10, 11].

\section{Clinical Trial}

Although clinical trial for efficacy might not be necessary for health food and supplements, we believe that herbal items in Chinese Medicine history with known biological effects could be better endorsed as health supplements with specific effects if the clinical responses to their consumption can be objectively proven.

A properly designed evidence-based clinical trial was adopted. A randomised, double-blind, placebo-control study aiming at post-menopausal women between $45-60$ years of age. 150 women were recruited and if their bone densities were lower than 
$0.894 \mathrm{gm} / \mathrm{cm} 2$, (i.e. osteopenic). They were allocated to receive either ELP or placebo for 12 months, 6 capsules per day. In ELP capsules, each contain 400mg ELP powder extract and 100mg calcium citrate; in placebo capsules, each contain color-matched starch and 100mg calcium citrate.

The end points included bone density (BMD), quality of life and adverse effects. The subjects were randomized into a treatment group and a placebo group. Data collection was organized at $0,4,8,12$ months.

\section{Results:}

In in-vitro studies, ELP significantly increased the proliferation of UMR-106 cells in dose-dependent manner from 50 to $400 \mathrm{ug} / \mathrm{ml}$ (Fig. 1). In MSCs, ELP stimulated the osteogenic differentiation as indicated by the increase of bone markers such as alkaline phosphatase (ALP) activity and matrix calcium mineralization. ELP elevated the ALP activity significantly at concentrations of 50 and $100 \mathrm{ug} / \mathrm{ml}$ (Fig. 2a). The amount of mineralization also increased significantly with ELP (Fig. 2b). In regards to bone resorption ELP significantly inhibited osteoclast formations from RAW 264.7 cells upon receptor activation of nuclear factor-kappaB ligand induction on the fourth day of treatment, at a concentration of 80 and $160 \mathrm{ug} / \mathrm{ml}$. The difference between treated and untreated cultures of osteoclasts reached statistical significance $(p \leq 0.05)$. Our experimental data therefore illustrated that the formula ELP had positive effects on bone metabolism through a double process of osteoblastic promotion and osteoclastic inhibition.

Figure 1. Effect of ELP water extract on cell viability of osteoblast-like UMR-106 cells for 3 days at different concentrations

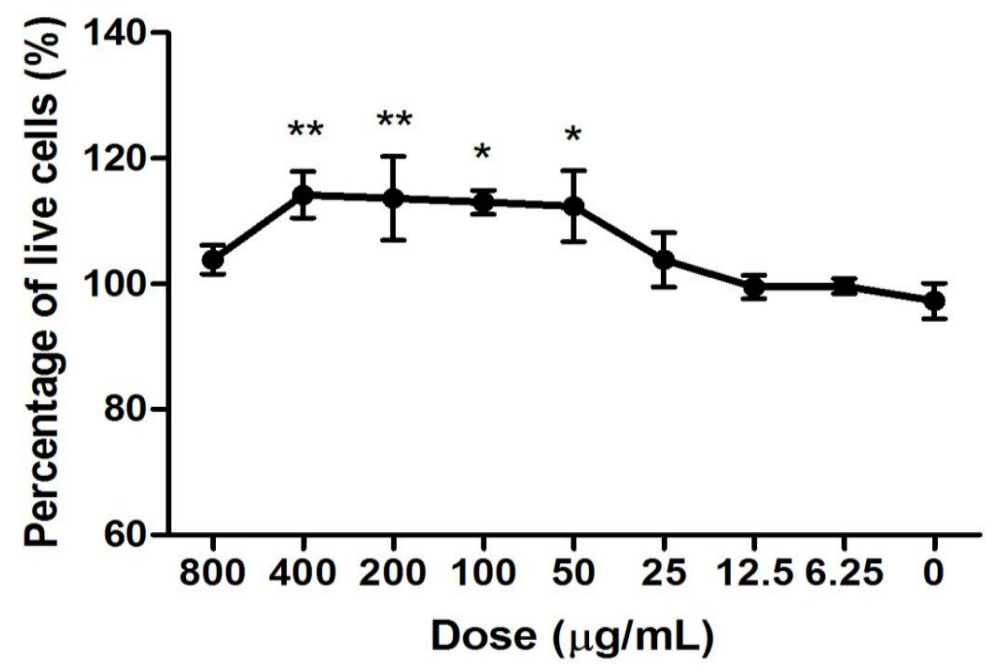


Data are the means (S.E.M; error bars) from three independent experiments in triplicate. * $p<0.05 ; * * p<0.01$ for difference in percentage of live cells from respective baseline culture without treatment

Figure 2a. Dose effect of ELP on the ALP activity in rat MSCs on day 7

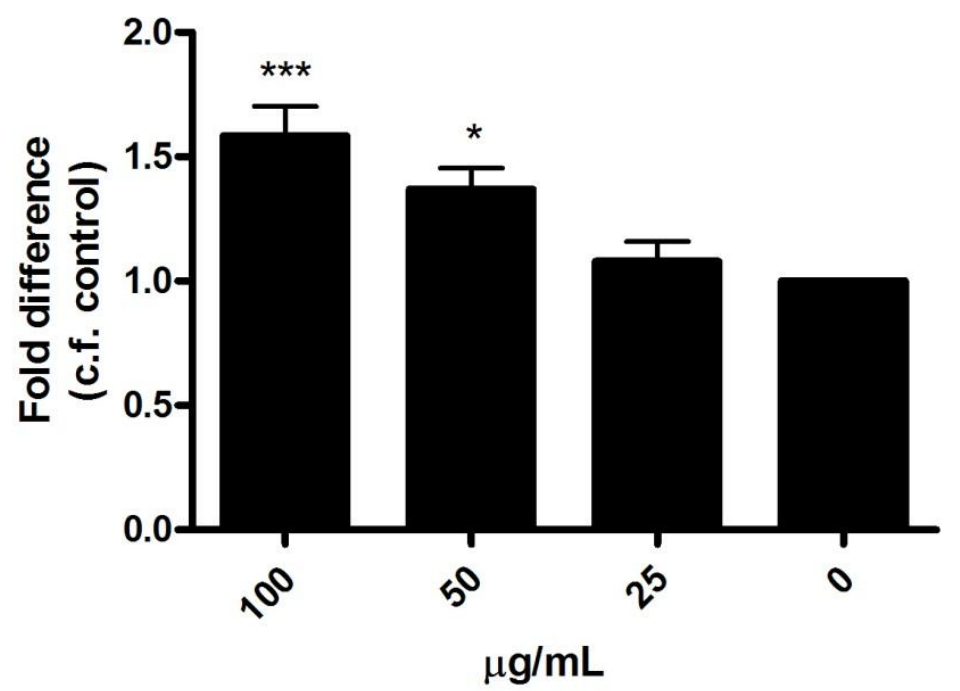

Figure 2b. Dose effect of ELP on the matrix mineralization in rat MSCs on day 7

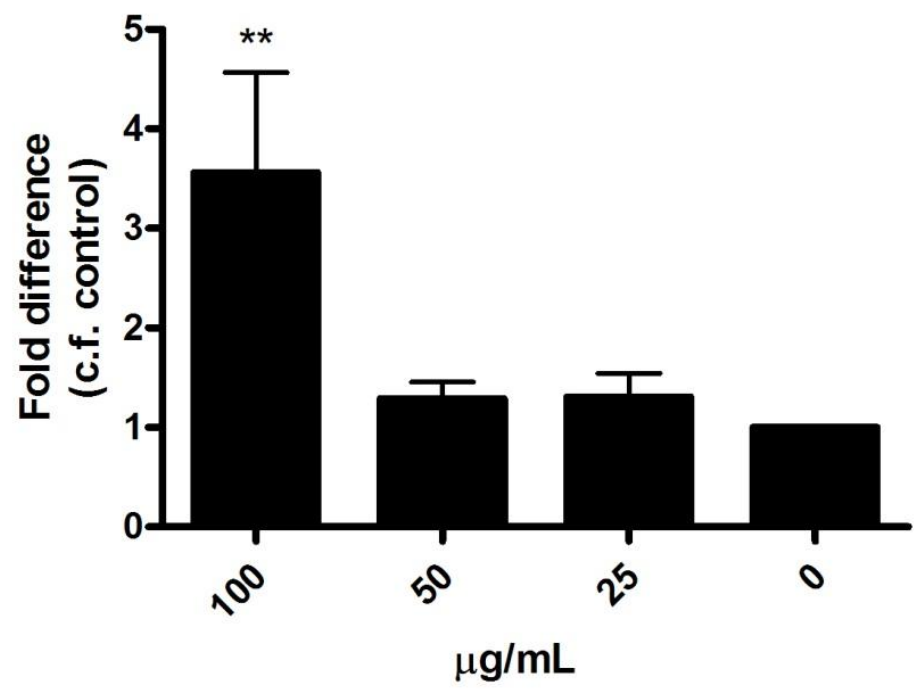

$* * * p<0.001 ; * * p<0.01 ; * p<0.05$

In ovariectomy-and calcium deficiency-induced osteoporotic rats model, after eleven weeks of ELP treatment, it demonstrated the beneficial effects on the preservation of bone mineral density at the proximal femur in a dose-dependent manner with the preference for higher dosage. No significant increase in uterus weight was observed in the ELP treated rats. In 
addition, microarray data of kidney tissue revealed that ELP was able to down-regulate the expression of phase II drug metabolizing enzymes, similar to the effects of estrogens.

In our clinical study, bboth treatment and placebo groups showed a decrease in spine BMD over 12 months treatment (ELP group $-0.31 \%$, placebo group $-0.39 \%$ ). However, the spine BMD of the ELP group was increased $0.69 \%$ in subjects who were more than 10 years after menopause, in contrast, the placebo group of the same stratum decreased by $0.61 \%$. The Hip BMD of the ELP group remained unchanged over 12-month treatment. While the overall hip BMD of the placebo group decreased by $0.22 \%$. In the subjects with more than 10 years' duration of menopause hip BMD increased by $0.21 \%$, whereas those treated with placebo decreased by $0.52 \%$ (Table 1 ).

Table 1. BMD changes between ELP and Placebo group.

\begin{tabular}{llll}
\hline & ELP & Placebo & $p$-value \\
\hline $\begin{array}{l}\text { Spine } \\
\text { Baseline, } \mathrm{g} / \mathrm{cm}^{2}\end{array}$ & $0.7994 \pm 0.1089$ & $0.7949 \pm 0.1011$ & 0.794 \\
$\quad$ Change from baseline \% & & & \\
$\quad$ At Month 6 & -0.01 & 0.35 & 0.432 \\
At Month 12 & -0.31 & -0.39 & 0.839
\end{tabular}

Subgroup: Menopausal duration >10 years
Baseline
$0.7996 \pm 0.1123$
$0.7547 \pm 0.1102$
0.176

Change from baseline $\%$

$\begin{array}{llll}\text { At Month 6 } & 0.13 & 0.18 & 0.984 \\ \text { At Month 12 } & 0.69 & -0.61 & 0.067\end{array}$

\section{Total hip}

Baseline, $\mathrm{g} / \mathrm{cm}^{2}$

$0.7573 \pm 0.0736$

$0.7592 \pm 0.0904$

0.885

Change from baseline $\%$

$\begin{array}{llll}\text { At Month } 6 & 0.19 & 0.44 & 0.379 \\ \text { At Month } 12 & 0.03 & -0.22 & 0.438\end{array}$

Subgroup: Menopausal duration $>10$ years

Baseline

$0.7492 \pm 0.0909$

$0.7296 \pm 0.0936$

0.473

Change from baseline $\%$

$\begin{array}{llll}\text { At Month 6 } & 0.63 & 0.30 & 0.516 \\ \text { At Month 12 } & 0.21 & -0.52 & 0.155\end{array}$


As regards the Spine BMD, subjects over 10 years after menopause showed a $0.7 \%$ increased after 12 months herbal treatment. In the placebo group, bone loss over 12 months reached $0.6 \%(\mathrm{p}=0.067)$.

The effects of the ELP treatment appeared to be quite different among two different groups of women stratified according to the duration after onset of menopause. Those having history of menopause for over 10 years were responding better to ELP: the gain in spine BMD was higher than that of the placebo group $(\mathrm{p}=0.028)$.

The trends of BMD changes at the Hip were similar to the spine but the difference between ELP and control groups did not attain statistical significance. If BMD represents the standard changes in the bone density, peripheral quantitative computed tomography (pQCT) which looks at the distal tibia and distal radius is more sensitive to early influences and rapid changes.

In this study, after 12 months of treatment using the ELP, the distal tibia pQCT decreased $2.43 \%$ in the ELP group compared with $3.67 \%$ in the placebo group $(\mathrm{p}=0.05)$ (Table 2). The tibia (T33\%) Strength-strain index (SSI) was increase 1.94\% in the ELP group compared with $0.33 \%$ in the placebo group $(\mathrm{p}=0.05)$ (Table 2$)$

Table 2. BMD changes at different anatomic sites measured by Peripheral Quantitative Computed Tomography (pQCT)

\begin{tabular}{lllllllll}
\hline Group & R4\% & R4\% & R4\% & T4\% & R4\% & R4\% & T4\% & T33\% \\
& Total & Trab & Cort & Trab & Total & Cort & Total & SSI \\
& BMD & BMD & BMD & BMD & BMD & BMD & BMD & \\
\hline ELP & $6.65 \%$ & $-1.99 \%$ & $3.57 \%$ & $-2.43 \%$ & $-0.74 \%$ & $3.65 \%$ & $6.15 \%$ & $1.94 \%$ \\
Placebo & $2.03 \%$ & $0.95 \%$ & $-0.28 \%$ & $-3.67 \%$ & $-2.04 \%$ & $-0.29 \%$ & $12.17 \%$ & $0.33 \%$ \\
\hline P value & & & 0.052 & & & & 0.047 \\
\hline
\end{tabular}

R4\% - Radius 4\% from distal end; T4\% - Tibia 4\% from distal end; T33\% - Tibia 33\% from distal end; Trab - Trabecular ; Cort- Cortical ; SSI- Strength Strain Index

\section{Quality of Life (QoL)}

The SF 36 QoL questionnaire was used of the eight domains included , physical function (PF), physical role (RP), bodily pain (BP), general health $(\mathrm{GH})$, vitality, social function, emotional role and mental health, only the four physical domains were evaluated. PF was remarkably improved compared with the baseline. Likewise the physical role (RP) was also improved. 


\section{Adverse Effects}

No significant adverse effects were encountered. Back pain, gastro-intestinal upset and common cold had been reported. Liver and renal functions were monitored with pre-and post-treatment blood checks. No significant changes were found.

\section{Discussion:}

Traditional Chinese Medicine is built on the principle of maintaining an internal harmony. Since specific pathological changes are unknown to the ancient experts, Chinese Medicine aims, not at specific targets, but at the maintenance of normal physiological balance. The well known theory of "yin and yang" is a philosophical expression of excess and deficiency which need to be balanced. In the case of osteoporosis prevention, when Chinese herbs are used, the rationale would be one of balanced bone formation and bone resorption. On a cellular level, it would be a balance between osteoblastic construction and osteoclastic destruction. The expected responses would be a steady BMD, and not a rapidly increasing one. When we plan a protocol on an osteoporosis prevention trial using Chinese herbs, the assessment target would need to follow accordingly, for example, good results would mean a BMD level that is not decreasing, but remains steady or rises slowly.

In Traditional Chinese Medicine 'osteoporosis', has not been described. However syndromes with low back pain, back deformities, loosening teeth etc. could be manifestations of osteoporosis. Chinese herbs have been widely used in orthopedic practice in ancient history. Many of their therapeutic effects in treating skeletal problems have been studied and reported in recent years [12 - 14]. Other Chinese herbs have potential effects on promoting fracture healing and bone health. In this study we have selected three common herbs from the pool of Chinese Medicine known to be good for bone health to form a cocktail. The three herbs have been used both as a traditional drug and as food items in soup making. There is no no adverse effect therefore these herbs are safe which has also been proven in our clinical trial. Use of these three herbs as a preventive agent for long term consumption would not raise any concern over safety.

In the clinical trial using the combined formula ELP, the trend of bone support is obvious as is shown in the steady and mild increase in the BMD across the different age groups although only those with more than ten years' menopause showed statistically significant improvements. The better results manifested in the proximal tibia which is more sensitive to interventions further illustrate the positive effects of the herbal formula.

\section{Conclusion:}


The three herbs formula ELP has strong support from Chinese medicine Classics as good agents for the maintenance of bone health. Our clinical trial using modern methodology has further offered evidence of their value. Now that potent anti-resorptive pharmaceuticals are showing more and more adverse effects, they might be suitable only for those suffering from severe osteoporosis, when there is immediate threat to fractures. Food supplements to maintain a good balance between bone formation and resorption could be timely offer for those who are osteopenic and only under the early threat of osteoporosis.

\section{Competing interests:}

All authors declare no conflicts of interest in this work.

\section{Author's contribution:}

Ping-chung Leung is the principle investigator for this study providing oversight and contributed fundamental conceptualization for the research and writing the draft manuscript. Erik Chun-hay Ko and Sammy Wing-sum Siu performed all of the lab work and conducted the statistical analysis for the animal study. Ellie Suet-yee Pang contributed to the research protocol and assisted in writing manuscript. King-fai Cheng contributed to the research protocol and conducted statistical analysis for the clinical study. Yat-heung Chan is the research coordinator for the clinical study.

\section{Acknowledgement:}

Authors are indebted to the Ming Lai Foundation and Hong Kong and Macau Tam Wah Ching Chinese Medicine Resource Centre support given to the team responsible for the study.

\section{References:}

1. Riggs BL, Melton LJ: The worldwide problem of osteoporosis: insights afforded by epidemiology. Bone 1995, 17(5 Suppl):505S-511S.

2. Dambacher MA, Schmitt S, Schacht E, Ito M, Neff M, Muller R, Qin L, Zhao YL: Bone structures in vitro and in vivo in animals and in men: a view into the future. J. Miner. Stoffwechs 2004, 11(3):13-19.

3. Nelson HD, Humphrey LL, Nygren P, Teutsch SM, Allan JD: Postmenopausal hormone replacement therapy: scientific review. JAMA 2002, 288(7):872-881.

4. Lau EMC: The Epidemiology of Osteoporosis in Asia. In Osteoporosis in Asia: crossing the frontiers. Edited by Lau EMC, Ho SC, Leung S, Woo J. Singapore: World Scientific; 1997: 1-20. 
5. Li NH, Ou PZ, Zhu HM, Yang DZ, Zheng PR, Liao EY: Epidemiological study on osteoporosis in middle-aged and elderly population in China. Chin. J. Clin. Rehabil. 2002, 6(5):758-759.

6. Cummings SR, Nevitt MC, Browner WS, Stone K, Fox KM, Ensrud KE, Cauley J, Black D, Vogt TM: Risk factors for hip fracture in white women. Study of Osteoporotic Fracutres Research Group. N. Engl. J. Med. 1995, 332(12):767-773.

7. Christiansen C: Prevention and treatment of osteoporosis with hormone replacement therapy. Int. J. Fertil. Menopausal Stud. 1993, 38(Suppl 1):45-54.

8. Consensus Development Conference: Diagnosis, Prophylaxis and treatment of osteoporosis. Am. J. Med. 1993, 94(6):646-650.

9. Lindsay R, Hart DM, Aitken JM, MacDonald EB, Anderson JB, Clarke AC: Long-term prevention of postmenopausal osteoporosis by oestrogen. Evidence for an increased bone mass after delay onset of oestrogen treatment. Lancet 1976, 1(7968): 1038-1041.

10. Qin L, Zhang G, Hung WY, Shi Y, Leung K, Yeung HY, Leung PC: Phytoestrogen-rich herb formula "XLGB" prevents OVX-induced deterioration of musculoskeletal tissues at the hip in old rats. J. Bone Miner. Metab. 2005, 23 (Suppl):55-61.

11. Zhang Y, Lai WP, Leung PC, Wu CF, Yao XS, Wong MS: Effects of Fructus Ligustri Lucidi extract on bone turnover and calcium balance in ovariectomized rats. Biol. Pharm. Bull. 2006, 29(2):291-296.

12. Yang F, Xu MR, Yang MF, Lam CH, Lau MT, Yuan ZH: Patterns of the use of traditional Chinese medicines in the treatment of osteoporosis. Chin. J. Clin. Rehabil. 2005, 9(31):203-205.

13. Pharmacopoeia Commission of the Ministry of Public Health: Kidney Tonics Chinese Pharmacopoeia Part I. Beijing China: Chemical Industrial Press; 2005.

14. Liang SM: Tonic Methods, Zhong Yao Fang Ji Xue. Guangdong China: Guangdong Science and Technology Press; 1991. 